\title{
Quantum polarization for three-dimensional fields via Stokes operators
}

\author{
Alfredo Luis* \\ Departamento de Óptica, Facultad de Ciencias Físicas, Universidad Complutense, 28040 Madrid, Spain
}

(Received 12 October 2004; published 17 February 2005)

\begin{abstract}
We study the polarization properties of three-dimensional quantum light fields by using the Stokes operators. We modify the standard definition of degree of polarization in order to encompass polarization properties in the quantum domain. We show that the states with the largest degree of polarization and least polarization fluctuations are the SU(3) coherent states. We show that the standard quadrature coherent states are Poissonian superpositions of $\mathrm{SU}(3)$ coherent states. We examine the polarization properties of some other relevant field states.
\end{abstract}

DOI: 10.1103/PhysRevA.71.023810

PACS number(s): 42.50.Dv, 03.65.Ca, 42.25.Ja

\section{INTRODUCTION}

Polarization is a fundamental ingredient of light, both in the quantum and in the classical domains. In the quantum regime this variable has been crucial in order to demonstrate experimentally fundamental properties and applications of the quantum theory such as entanglement, complementarity, quantum cryptography, teleportation, and Bell inequalities [1] and also concerning the proper quantum description of the phase [2].

Although the electric field is by definition a threedimensional (3D) magnitude, the polarization of strictly harmonic classical waves is locally a two-dimensional (2D) phenomenon, since the electric field at each spatial point describes an ellipse contained in a plane. Nevertheless, the plane of the ellipse may vary from point to point so that a three-dimensional analysis may be useful, especially for nonparaxial beams without a well-defined propagation direction.

Moreover, quantum fluctuations affect the three field components even when they are in the vacuum state, so that for every field state the quantum electric field varies unavoidably in a $3 \mathrm{D}$ region [3]. Therefore, in the quantum domain polarization is always a 3D phenomenon.

The 3D description of polarization has been already addressed in the classical domain by a suitable generalization of the Stokes parameters derived from the $3 \times 3$ coherence matrix [4-10].

In this work we address a quantum description of $3 \mathrm{D}$ polarization by using the 3D Stokes operators [11]. In Sec. II we recall the basic definitions. In Secs. III and IV we show that the states with the largest degree of 3D polarization and the least polarization fluctuations are the SU(3) coherent states. In Sec. V we show that standard quadrature coherent states are Poissonian superpositions of SU(3) coherent states, which in turn are superpositions of SU(2) coherent states. In Sec. VI we examine the polarization properties of some other relevant field states. In Sec. VII we study the superposition of partially polarized light into completely polarized and completely unpolarized components.

\footnotetext{
*Electronic address: alluis@ fis.ucm.es; URL: http://www.ucm.es/ info/gioq
}

\section{STOKES OPERATORS AND DEGREE OF POLARIZATION}

The standard two-mode Stokes parameters can be generalized to a three-mode field in the form [4,6-10]

$$
\left\langle S_{j}\right\rangle=\operatorname{tr}\left(\lambda_{j} \Phi\right)
$$

$j=0,1, \ldots, 8$, where $\Phi$ is the $3 \times 3$ coherence matrix

$$
\Phi_{k, \ell}=\left\langle a_{\ell}^{\dagger} a_{k}\right\rangle,
$$

$k, \ell=1,2,3 a_{\ell}$ being the complex amplitudes and $\lambda_{j}$ the nine Gell-Mann matrices [the generators of the SU(3) group]

$$
\begin{aligned}
& \lambda_{0}=\left(\begin{array}{lll}
1 & 0 & 0 \\
0 & 1 & 0 \\
0 & 0 & 1
\end{array}\right) \\
& \lambda_{1}=\left(\begin{array}{lll}
0 & 1 & 0 \\
1 & 0 & 0 \\
0 & 0 & 0
\end{array}\right), \lambda_{2}=\left(\begin{array}{ccc}
0 & -i & 0 \\
i & 0 & 0 \\
0 & 0 & 0
\end{array}\right), \\
& \lambda_{3}=\left(\begin{array}{ccc}
1 & 0 & 0 \\
0 & -1 & 0 \\
0 & 0 & 0
\end{array}\right), \lambda_{4}=\left(\begin{array}{lll}
0 & 0 & 1 \\
0 & 0 & 0 \\
1 & 0 & 0
\end{array}\right), \\
& \lambda_{5}=\left(\begin{array}{ccc}
0 & 0 & -i \\
0 & 0 & 0 \\
i & 0 & 0
\end{array}\right), \lambda_{6}=\left(\begin{array}{ccc}
0 & 0 & 0 \\
0 & 0 & 1 \\
0 & 1 & 0
\end{array}\right) \text {, } \\
& \lambda_{7}=\left(\begin{array}{ccc}
0 & 0 & 0 \\
0 & 0 & -i \\
0 & i & 0
\end{array}\right), \lambda_{8}=\frac{1}{\sqrt{3}}\left(\begin{array}{ccc}
1 & 0 & 0 \\
0 & 1 & 0 \\
0 & 0 & -2
\end{array}\right),
\end{aligned}
$$

which verify the trace-orthogonality relations

$$
\operatorname{tr}\left(\lambda_{j} \lambda_{k}\right)=2 \delta_{j, k}+\delta_{j, 0} \delta_{k, 0} .
$$

The above relation (2.1) can be inverted in the form 


$$
\Phi=\frac{1}{2}\left(\begin{array}{ccc}
\frac{2}{3}\left\langle S_{0}\right\rangle+\left\langle S_{3}\right\rangle+\frac{1}{\sqrt{3}}\left\langle S_{8}\right\rangle & \left\langle S_{1}\right\rangle-i\left\langle S_{2}\right\rangle & \left\langle S_{4}\right\rangle-i\left\langle S_{5}\right\rangle \\
\left\langle S_{1}\right\rangle+i\left\langle S_{2}\right\rangle & \frac{2}{3}\left\langle S_{0}\right\rangle-\left\langle S_{3}\right\rangle+\frac{1}{\sqrt{3}}\left\langle S_{8}\right\rangle & \left\langle S_{6}\right\rangle-i\left\langle S_{7}\right\rangle \\
\left\langle S_{4}\right\rangle+i\left\langle S_{5}\right\rangle & \left\langle S_{6}\right\rangle+i\left\langle S_{7}\right\rangle & \frac{2}{3}\left\langle S_{0}\right\rangle-\frac{2}{\sqrt{3}}\left\langle S_{8}\right\rangle
\end{array}\right) .
$$

The coherence matrix and the Stokes parameters provide exactly the same information. The motivation for using the Stokes parameters comes from the fact that they are real measurable quantities that can be determined via standard experimental procedures [6]. Furthermore, for twodimensional fields they provide a very illustrative and useful picture of polarization via the Poincaré sphere (although its generalization to three-dimensional fields provides lesser geometrical intuition due to the increase of the dimension).

In the quantum domain, the Stokes parameters are the mean values of the Stokes operators [11]

$$
S_{j}=\mathbf{a}^{\dagger} \lambda_{j} \mathbf{a},
$$

for $j=0,1, \ldots, 8$, where $\mathbf{a}$ and $\mathbf{a}^{\dagger}$ are three-dimensional column and row vectors made of the complex amplitude operators and their Hermitian conjugates, respectively.

The generalized Stokes parameters allow us to introduce a $3 \mathrm{D}$ degree of polarization in the form $[4,6-9]$

$$
P_{3 \mathrm{D}}=\frac{\sqrt{3}}{2} \frac{\sqrt{\sum_{j=1}^{8}\left\langle S_{j}\right\rangle^{2}}}{\left\langle S_{0}\right\rangle} .
$$

To some extent, this definition does not grasp the limits to polarization established by quantum fluctuations. Since the Stokes operators do not commute, no field state can have definite values of all of them simultaneously, so every state presents polarization fluctuations. In other words, no quantum state has a definite polarization ellipse for the same reasons that quantum particles do not follow definite classical trajectories. The definition (2.7) does not reflect this idea, since there are field states with $P_{3 \mathrm{D}}=1$ (for example, it is shown below that this is the case of quadrature coherent states). Moreover, this occurs even for field states as close as desired to the three-dimensional vacuum state, which is a fully unpolarized state according to every criterion.

There is a simple modification of the above definition that avoids this difficulty as a generalization to three dimensions of the two-dimensional proposal in Ref. [12],

$$
P_{3 \mathrm{D}}=\frac{\sqrt{\sum_{j=1}^{8}\left\langle S_{j}\right\rangle^{2}}}{\sqrt{\sum_{j=1}^{8}\left\langle S_{j}^{2}\right\rangle}}=\frac{\sqrt{3}}{2} \frac{\sqrt{\sum_{j=1}^{8}\left\langle S_{j}\right\rangle^{2}}}{\sqrt{\left\langle S_{0}\left(S_{0}+3\right)\right\rangle}},
$$

where we have used the operator identity

$$
\sum_{k=1}^{8} S_{k}^{2}=\sum_{k=1}^{8} \sum_{i, j, \ell, m=1}^{3} \lambda_{k, i j} \lambda_{k, \ell m} a_{i}^{\dagger} a_{j} a_{\ell}^{\dagger} a_{m}=\frac{4}{3} S_{0}\left(S_{0}+3\right) .
$$

It is worth noting that this definition seemingly depends on the fluctuations of the intensity $\Delta S_{0}$ since in the denominator

$$
\left\langle S_{0}^{2}\right\rangle=\left(\Delta S_{0}\right)^{2}+\left\langle S_{0}\right\rangle^{2} .
$$

At first sight this dependence might be regarded as a drawback, because such a dependence is absent in the classical domain. Nevertheless, in the next section we show that this dependence is necessary in the quantum domain provided that we naturally link the maximum degree of polarization with minimum fluctuations of the Stokes operators, a relation that is not fulfilled when using Eq. (2.7).

Leaving aside these remarks, in most applications both expressions (2.7) and (2.8) are interchangeable since their differences are minimal, especially when approaching the classical limit where they coincide.

\section{MAXIMALLY POLARIZED STATES}

In this section we look for three-dimensional field states with the largest degree of polarization. In order to maximize Eqs. (2.7) and (2.8), we begin by computing their common numerator

$$
\begin{aligned}
\sum_{k=1}^{8}\left\langle S_{k}\right\rangle^{2}= & \frac{4}{3}\left(\left\langle a_{1}^{\dagger} a_{1}\right\rangle^{2}+\left\langle a_{2}^{\dagger} a_{2}\right\rangle^{2}+\left\langle a_{3}^{\dagger} a_{3}\right\rangle^{2}-\left\langle a_{1}^{\dagger} a_{1}\right\rangle\left\langle a_{2}^{\dagger} a_{2}\right\rangle\right. \\
& -\left\langle a_{1}^{\dagger} a_{1}\right\rangle\left\langle a_{3}^{\dagger} a_{3}\right\rangle-\left\langle a_{2}^{\dagger} a_{2}\right\rangle\left\langle a_{3}^{\dagger} a_{3}\right\rangle+3\left|\left\langle a_{1}^{\dagger} a_{2}\right\rangle\right|^{2} \\
& \left.+3\left|\left\langle a_{1}^{\dagger} a_{3}\right\rangle\right|^{2}+3\left|\left\langle a_{2}^{\dagger} a_{3}\right\rangle\right|^{2}\right),
\end{aligned}
$$

where we have used that the only terms different from zero in the $k$ sum are

$$
\begin{gathered}
\sum_{k=1}^{8} \lambda_{k, i i} \lambda_{k, i i}=\frac{4}{3}, \\
\sum_{k=1}^{8} \lambda_{k, i i} \lambda_{k, j j}=-\frac{2}{3}, i \neq j, \\
\sum_{k=1}^{8} \lambda_{k, i j} \lambda_{k, j i}=2 .
\end{gathered}
$$

We proceed by using the Cauchy-Schwarz inequalities

$$
\left|\left\langle a_{i}^{\dagger} a_{j}\right\rangle\right|^{2} \leqslant\left\langle a_{i}^{\dagger} a_{i}\right\rangle\left\langle a_{j}^{\dagger} a_{j}\right\rangle,
$$

leading to 


$$
\sum_{k=1}^{8}\left\langle S_{k}\right\rangle^{2} \leqslant \frac{4}{3}\left\langle S_{0}\right\rangle^{2}
$$

The maximum is obtained when all the Cauchy-Schwarz relations are equalities, which occurs provided that

$$
a_{1}|\psi\rangle \propto a_{2}|\psi\rangle \propto a_{3}|\psi\rangle .
$$

In the next section we show that for a fixed total number $S_{0}$ this is satisfied only by the $\mathrm{SU}(3)$ coherent states as demonstrated in Eq. (4.8) below.

Equation (3.4) implies that for definition (2.7) we get $P_{3 \mathrm{D}} \leqslant 1$, irrespectively of the value of $\left\langle S_{0}\right\rangle$. The equality is reached by all the states fulfilling Eq. (3.5). This is the case of SU(3) coherent states and quadrature coherent states for example.

On the other hand, for the definition (2.8) we can use Eq. (3.4) in the numerator and Eq. (2.10) in the denominator (which implies $\left\langle S_{0}^{2}\right\rangle \geqslant\left\langle S_{0}\right\rangle^{2}$, the equality being reached when $\left.\Delta S_{0}=0\right)$, leading to

$$
P_{3 \mathrm{D}} \leqslant \frac{1}{\sqrt{1+\frac{3}{\left\langle S_{0}\right\rangle}}}
$$

In this case the maximum degree of polarization attainable depends on the intensity of the wave through the value of $\left\langle S_{0}\right\rangle$. The equality is reached by the eigenstates of $S_{0}$ fulfilling Eq. (3.5). These are just the SU(3) coherent states exclusively.

Finally, we note that it would be natural to expect that maximum degree of polarization should be equivalent to minimum fluctuations of the Stokes operators. This identification can be validated if we adopt a suitable measure of the global fluctuations of the eight Stokes operators. In this regard, the most appropriate measure for our purposes is [13]

$$
\sum_{k=1}^{8}\left(\Delta S_{k}\right)^{2}=\sum_{k=1}^{8}\left\langle S_{k}^{2}\right\rangle-\sum_{k=1}^{8}\left\langle S_{k}\right\rangle^{2}=\frac{4}{3}\left\langle S_{0}\left(S_{0}+3\right)\right\rangle-\sum_{k=1}^{8}\left\langle S_{k}\right\rangle^{2} .
$$

From Eqs. (2.10), (3.7), and (3.4) we have

$$
\sum_{k=1}^{8}\left(\Delta S_{k}\right)^{2} \geqslant 4\left\langle S_{0}\right\rangle
$$

the equality being reached provided that $\Delta S_{0}=0$ and that Eq. (3.5) holds.

We can see that minimum polarization fluctuations are equivalent to a maximum degree of polarization only when using Eq. (2.8). This is not the case when using definition (2.7), since the quadrature coherent states, for example, present fluctuations of the Stokes operators well above the minimum [see Eq. (7.7)].

\section{SU(3) COHERENT STATES}

The SU(3) coherent state $|N, \Gamma\rangle$ can be defined by the action of a $\mathrm{SU}(3)$ transformation on the number state $|0,0, N\rangle[14]$,

$$
|N, \Gamma\rangle=e^{i a^{\dagger} R a}|0,0, N\rangle,
$$

where $R$ is a Hermitian $3 \times 3$ matrix, $\Gamma$ represents the parameters required to suitably specify $R$, and $|0,0, N\rangle$ is the product of the vacuum state in modes $a_{1}, a_{2}$ and the number state $N$ in mode $a_{3}$. The above transformation acting on $|0,0, N\rangle$ can be expressed also as a product of two consecutive $\mathrm{SU}(2)$ transformations:

$$
|N, \Gamma\rangle=e^{\theta\left(e^{-i \phi} a_{2}^{\dagger} a_{1}-e^{i \phi} a_{1}^{\dagger} a_{2}\right) / 2} e^{\theta^{\prime}\left(e^{-i \phi^{\prime}} a_{3}^{\dagger} a_{2}-e^{i \phi^{\prime}} a_{2}^{\dagger} a_{3}\right) / 2}|0,0, N\rangle .
$$

This produces the following transformation of the complex amplitude operators:

$$
e^{-i a^{\dagger} R a} a e^{i a^{\dagger} R a}=\mathcal{U} a,
$$

where $\mathcal{U}$ is the unitary transformation [15],

$$
\mathcal{U}=\left(\begin{array}{ccc}
c & -s e^{i \phi} & 0 \\
s e^{-i \phi} & c & 0 \\
0 & 0 & 1
\end{array}\right)\left(\begin{array}{ccc}
1 & 0 & 0 \\
0 & c^{\prime} & -s^{\prime} e^{i \phi^{\prime}} \\
0 & s^{\prime} e^{-i \phi^{\prime}} & c^{\prime}
\end{array}\right),
$$

being $c=\cos (\theta / 2), s=\sin (\theta / 2)$, and similarly for the primed variables.

These states are eigenstates of the total number operator

$$
S_{0}|N, \Gamma\rangle=N|N, \Gamma\rangle \text {. }
$$

Furthermore, we can demonstrate that

$$
a_{1}|N, \Gamma\rangle \propto a_{2}|N, \Gamma\rangle \propto a_{3}|N, \Gamma\rangle .
$$

This is because

$$
a_{j} e^{i a^{\dagger} R a}|0,0, N\rangle=e^{i a^{\dagger} R a} \sum_{k=1}^{3} \mathcal{U}_{j, k} a_{k}|0,0, N\rangle=\mathcal{U}_{j, 3} e^{i a^{\dagger} R a} a_{3}|0,0, N\rangle,
$$

so that

$$
\frac{1}{\mathcal{U}_{1,3}} a_{1}|N, \Gamma\rangle=\frac{1}{\mathcal{U}_{2,3}} a_{2}|N, \Gamma\rangle=\frac{1}{\mathcal{U}_{3,3}} a_{3}|N, \Gamma\rangle .
$$

According to the results of the preceding section, Eqs. (4.5) and (4.6) demonstrate that the SU(3) coherent states have minimum polarization fluctuations and maximum degree of polarization.

Vice versa, all the states satisfying Eq. (4.8) within each subspace of fixed total photon number $S_{0}$ are SU(3) coherent states. This is because the consecutive application of two suitable SU(2) transformations changes Eq. (4.8) into

$$
a_{1}|\psi\rangle=a_{2}|\psi\rangle=0
$$

whose unique solution in each subspace of fixed total number $N$ is the number state $|0,0, N\rangle$.

From Eqs. (4.1), (4.3), and (4.4) the Stokes parameters for $|N, \Gamma\rangle$ are

$$
\left\langle S_{k}\right\rangle=N\left(\mathcal{U}^{\dagger} \lambda_{k} \mathcal{U}\right)_{3,3}=N \mu_{k},
$$

for $k=0,1, \ldots, 8$, where the functions $\mu_{k}$ are 


$$
\begin{aligned}
& \mu_{0}=1 \text {, } \\
& \mu_{1}=2 \sin ^{2} \frac{\theta^{\prime}}{2} \sin \frac{\theta}{2} \cos \frac{\theta}{2} \cos \phi, \\
& \mu_{2}=2 \sin ^{2} \frac{\theta^{\prime}}{2} \sin \frac{\theta}{2} \cos \frac{\theta}{2} \sin \phi, \\
& \mu_{3}=\sin ^{2} \frac{\theta^{\prime}}{2}\left(\sin ^{2} \frac{\theta}{2}-\cos ^{2} \frac{\theta}{2}\right), \\
& \mu_{4}=2 \cos \frac{\theta^{\prime}}{2} \sin \frac{\theta^{\prime}}{2} \sin \frac{\theta}{2} \cos \left(\phi+\phi^{\prime}\right), \\
& \mu_{5}=2 \cos \frac{\theta^{\prime}}{2} \sin \frac{\theta^{\prime}}{2} \sin \frac{\theta}{2} \sin \left(\phi+\phi^{\prime}\right), \\
& \mu_{6}=2 \cos \frac{\theta^{\prime}}{2} \sin \frac{\theta^{\prime}}{2} \cos \frac{\theta}{2} \cos \phi^{\prime}, \\
& \mu_{7}=2 \cos \frac{\theta^{\prime}}{2} \sin \frac{\theta^{\prime}}{2} \cos \frac{\theta}{2} \sin \phi^{\prime}, \\
& \mu_{8}=\frac{1}{\sqrt{3}}\left(\sin ^{2} \frac{\theta^{\prime}}{2}-2 \cos ^{2} \frac{\theta^{\prime}}{2}\right) .
\end{aligned}
$$

It can be checked that $\sum_{k=1}^{8}\left\langle S_{k}\right\rangle^{2}=(4 / 3)\left\langle S_{0}\right\rangle^{2}$.

\section{NESTED COHERENT STATES}

In this section we show that there is an interesting relationship between quadrature coherent states, SU(3) coherent states, $\mathrm{SU}(2)$ coherent states, and number states.

We begin by considering a product of quadrature coherent states in each mode $|\boldsymbol{\alpha}\rangle=\left|\alpha_{1}\right\rangle\left|\alpha_{2}\right\rangle\left|\alpha_{3}\right\rangle$ with $a_{j}|\boldsymbol{\alpha}\rangle=\alpha_{j}|\boldsymbol{\alpha}\rangle$. Expressing these vectors in the number basis we get

$$
|\boldsymbol{\alpha}\rangle=e^{-r^{2} / 2} \sum_{N=0}^{\infty} \frac{\left(r e^{i \delta}\right)^{N}}{\sqrt{N !}}|N, \Gamma\rangle,
$$

where $|N, \Gamma\rangle$ are $\mathrm{SU}(3)$ coherent states [14],

$$
\begin{aligned}
|N, \Gamma\rangle= & \sum_{n=0}^{N}\left(\begin{array}{c}
N \\
n
\end{array}\right)^{1 / 2}\left(\cos \frac{\theta^{\prime}}{2}\right)^{N-n}\left(\sin \frac{\theta^{\prime}}{2}\right)^{n} \\
& \times e^{-i n \phi^{\prime}}|n, \Omega\rangle_{1,2}|N-n\rangle_{3},
\end{aligned}
$$

$|n, \Omega\rangle_{1,2}$ are $\mathrm{SU}(2)$ coherent states in modes $a_{1}, a_{2}$ [16],

$$
|n, \Omega\rangle_{1,2}=\sum_{m=0}^{n}\left(\begin{array}{l}
n \\
m
\end{array}\right)^{1 / 2}\left(\cos \frac{\theta}{2}\right)^{n-m}\left(\sin \frac{\theta}{2}\right)^{m} e^{-i m \phi}|m\rangle_{1}|n-m\rangle_{2},
$$

and $|m\rangle_{1},|n-m\rangle_{2}$, and $|N-n\rangle_{3}$ are number states in the corresponding modes. The angular variables above are related to the complex amplitudes $\boldsymbol{\alpha}$ in the form

$$
\begin{gathered}
\alpha_{1}=r \sin \frac{\theta^{\prime}}{2} \sin \frac{\theta}{2} e^{i \delta} e^{-i \phi^{\prime}} e^{-i \phi}, \\
\alpha_{2}=r \sin \frac{\theta^{\prime}}{2} \cos \frac{\theta}{2} e^{i \delta} e^{-i \phi^{\prime}}, \\
\alpha_{3}=r \cos \frac{\theta^{\prime}}{2} e^{i \delta},
\end{gathered}
$$

where $\infty \geqslant r \geqslant 0, \pi \geqslant \theta, \theta^{\prime} \geqslant 0$, and $2 \pi \geqslant \phi, \phi^{\prime} \geqslant 0$.

In Eqs. (5.1)-(5.3) we can recognize an interesting structure. The three-mode quadrature coherent state $|\boldsymbol{\alpha}\rangle$ is formally identical to a one-mode coherent state, but replacing the number states $|N\rangle$ by the $\mathrm{SU}(3)$ coherent states $|N, \Gamma\rangle$ (which are eigenstates of the total number operator $a_{1}^{\dagger} a_{1}$ $+a_{2}^{\dagger} a_{2}+a_{3}^{\dagger} a_{3}$ with eigenvalue $\left.N\right)$. In turn, $|N, \Gamma\rangle$ has the same structure of a two-mode $\mathrm{SU}(2)$ coherent state but replacing the number states $|n\rangle$ in one of the modes by the two-mode $\mathrm{SU}(2)$ coherent states $|n, \Omega\rangle_{1,2}$ (which are eigenstates of the number of photons $a_{1}^{\dagger} a_{1}+a_{2}^{\dagger} a_{2}$ with eigenvalue $n$ ), as shown in Ref. [14].

It is clear that the role played by each one of the modes in this chain of coherent states can be interchanged. Some other examples of this grouping of coherent states can be found in Ref. [17].

\section{RELATION BETWEEN THE 3D AND 2D DEGREES OF POLARIZATION}

It is natural to ask whether there exists a definite relation between two- and three-dimensional approaches to quantum polarization. This is because we can imagine every twodimensional field as being three dimensional with a vanishing component. Therefore, to every two-dimensional field we can assign two different degrees of polarization $P_{3 \mathrm{D}}$ and $P_{2 \mathrm{D}}$, which need not coincide necessarily.

In this regard, the field states as close as possible to have a vanishing component—say, $a_{3}$ —are obtained by assuming that the mode $a_{3}$ is in the vacuum state,

$$
\rho_{3 \mathrm{D}}=\rho_{2 \mathrm{D}} \otimes|0\rangle_{33}\langle 0|,
$$

where $|0\rangle_{3}$ is the vacuum state in mode $a_{3}$. In such a case we have the following relation between the three-dimensional and two-dimensional values of the Stokes parameters:

$$
\sum_{k=1}^{8}\left\langle S_{k}\right\rangle_{3 \mathrm{D}}^{2}=\sum_{k=1}^{3}\left\langle S_{k}\right\rangle_{2 \mathrm{D}}^{2}+\frac{1}{3}\left\langle S_{0}\right\rangle^{2}
$$

where in the $2 \mathrm{D}$ case

$$
\begin{aligned}
& \left\langle S_{1}\right\rangle_{2 \mathrm{D}}=\left\langle\left(a_{1}^{\dagger} a_{2}+a_{2}^{\dagger} a_{1}\right)\right\rangle, \\
& \left\langle S_{2}\right\rangle_{2 \mathrm{D}}=\left\langle i\left(a_{2}^{\dagger} a_{1}-a_{1}^{\dagger} a_{2}\right)\right\rangle, \\
& \left\langle S_{3}\right\rangle_{2 \mathrm{D}}=\left\langle\left(a_{1}^{\dagger} a_{1}-a_{2}^{\dagger} a_{2}\right)\right\rangle,
\end{aligned}
$$

being $\left\langle S_{0}\right\rangle=\left\langle S_{0}\right\rangle_{3 \mathrm{D}}=\left\langle S_{0}\right\rangle_{2 \mathrm{D}}$.

If we were using the standard definition (2.7) we would get the general relation 


$$
P_{3 \mathrm{D}}^{2}=\frac{3}{4} P_{2 \mathrm{D}}^{2}+\frac{1}{4},
$$

where $P_{2 \mathrm{D}}$ is the 2D analog of Eq. (2.7).

We always have $P_{3 \mathrm{D}} \geqslant P_{2 \mathrm{D}}$, the equality being reached only for $P_{2 \mathrm{D}}=1$. A clear example of the case $P_{3 \mathrm{D}}>P_{2 \mathrm{D}}$ occurs when $P_{2 \mathrm{D}}=0$, for which $P_{3 \mathrm{D}}=1 / 2$. This result is consistent since fully unpolarized two-dimensional light (two random uncorrelated components of the same intensity) is certainly not completely unpolarized in three dimensions (three random uncorrelated components of the same intensity) [8].

On the other hand, from Eq. (2.8) and using as well its two-dimensional counterpart [12]

$$
P_{2 \mathrm{D}}^{2}=\frac{\sum_{k=1}^{3}\left\langle S_{k}\right\rangle_{2 \mathrm{D}}^{2}}{\left\langle S_{0}^{2}\right\rangle+2\left\langle S_{0}\right\rangle},
$$

we get

$$
P_{3 \mathrm{D}}^{2}=\frac{3\left(\left\langle S_{0}^{2}\right\rangle+2\left\langle S_{0}\right\rangle\right) P_{2 \mathrm{D}}^{2}+\left\langle S_{0}\right\rangle^{2}}{4\left(\left\langle S_{0}^{2}\right\rangle+3\left\langle S_{0}\right\rangle\right)} .
$$

In this case and contrary to Eq. (6.4), we have the possibility of $P_{3 \mathrm{D}}<P_{2 \mathrm{D}}$. This occurs provided that

$$
P_{2 \mathrm{D}}^{2}>\frac{\left\langle S_{0}\right\rangle^{2}}{\left\langle S_{0}^{2}\right\rangle+6\left\langle S_{0}\right\rangle} .
$$

For the sake of illustration let us consider the maximum $P_{2 \mathrm{D}}$ allowed by Eq. (6.5) for $\left\langle S_{0}^{2}\right\rangle=\left\langle S_{0}\right\rangle^{2}=1$. In this case we have $P_{2 \mathrm{D}}=0.58$ while $P_{3 \mathrm{D}}=0.50$.

\section{SOME EXAMPLES}

Next we apply the above definitions to some relevant field states.

\section{A. One-photon states}

The Hilbert space for one photon split between three modes is spanned by the photon-number states $|1\rangle=|1,0,0\rangle$, $|2\rangle=|0,1,0\rangle$, and $|3\rangle=|0,0,1\rangle$. Taking this as a basis, it can be seen that the coherence matrix $\Phi$ and the density matrix $\rho$ coincide,

$$
\langle\ell|\rho| k\rangle=\left\langle a_{k}^{\dagger} a_{\ell}\right\rangle=\Phi_{\ell, k},
$$

and $\rho$ admits the expression (2.5).

Moreover, every one-photon pure state is a $\mathrm{SU}(3)$ coherent state

$$
\begin{aligned}
|1, \Gamma\rangle= & \cos \frac{\theta^{\prime}}{2}|0,0,1\rangle+\cos \frac{\theta}{2} \sin \frac{\theta^{\prime}}{2} e^{-i \phi^{\prime}}|0,1,0\rangle \\
& +\sin \frac{\theta}{2} \sin \frac{\theta^{\prime}}{2} e^{-i\left(\phi+\phi^{\prime}\right)}|1,0,0\rangle .
\end{aligned}
$$

Therefore, every pure state has maximum degree of polarization and minimum polarization fluctuations for $\left\langle S_{0}\right\rangle=1$.

\section{B. Number states}

For the number states $\left|n_{1}, n_{2}, n_{3}\right\rangle$ the only nonvanishing Stokes parameters are

$$
\begin{gathered}
\left\langle S_{0}\right\rangle=n_{1}+n_{2}+n_{3}, \\
\left\langle S_{3}\right\rangle=n_{1}-n_{2}, \\
\left\langle S_{8}\right\rangle=\frac{1}{\sqrt{3}}\left(n_{1}+n_{2}-2 n_{3}\right),
\end{gathered}
$$

leading to

$$
\sum_{k=1}^{8}\left\langle S_{k}\right\rangle^{2}=2\left(n_{1}^{2}+n_{2}^{2}+n_{3}^{2}\right)-\frac{2}{3}\left(n_{1}+n_{2}+n_{3}\right)^{2} .
$$

For the equipartition state $n_{1}=n_{2}=n_{3}=\left\langle S_{0}\right\rangle / 3$ all the Stokes parameters vanish (except $\left\langle S_{0}\right\rangle$ ), so that this state reaches the minimum degree of polarization $P_{3 \mathrm{D}}=0$ and the maximum fluctuations of the Stokes parameters:

$$
\sum_{k=1}^{8}\left(\Delta S_{k}\right)^{2}=\frac{4}{3}\left\langle S_{0}\right\rangle\left(\left\langle S_{0}\right\rangle+3\right) .
$$

The other extreme situation occurs when all the photons are concentrated in a single mode-for example, $n_{1}=n_{2}=0$, $n_{3} \neq 0$. This state is actually a $\mathrm{SU}(3)$ coherent state with $\theta$ $=\theta^{\prime}=0$, reaching a maximum degree of polarization and minimum polarization fluctuations.

These conclusions are equally valid irrespective of whether we use Eq. (2.7) or Eq. (2.8).

\section{Quadrature coherent states}

As we have mentioned above the quadrature coherent states are Poissonian superpositions of SU(3) coherent states. This suggests that they should be close to have a maximum degree of polarization and minimum polarization uncertainty.

They reach the maximum value allowed for the sum of the squares of the Stokes parameters in Eq. (3.4),

$$
\sum_{k=1}^{8}\left\langle S_{k}\right\rangle^{2}=\frac{4}{3}\left\langle S_{0}\right\rangle^{2},
$$

since they satisfy conditions (3.5) trivially.

However, they are not exactly extremal concerning polarization fluctuations because of the fluctuations of the total number of photons $\left(\Delta S_{0}\right)^{2}=\left\langle S_{0}\right\rangle$ so that

$$
\sum_{k=1}^{8}\left(\Delta S_{k}\right)^{2}=\frac{16}{3}\left\langle S_{0}\right\rangle
$$

which exceeds by $33 \%$ the minimum $4\left\langle S_{0}\right\rangle$.

As we have discussed above this is reflected or not by the degree of polarization depending on the definition used. When using Eq. (2.7) we have a maximum degree $P_{3 \mathrm{D}}=1$ for all $\left\langle S_{0}\right\rangle$, while when using Eq. (2.8) 


$$
P_{3 \mathrm{D}}=\frac{1}{\sqrt{1+\frac{4}{\left\langle S_{0}\right\rangle}}},
$$

which is not the maximum in Eq. (3.6), but is nevertheless very close to it when $\left\langle S_{0}\right\rangle$ is large enough.

\section{POLARIZED-UNPOLARIZED LIGHT DECOMPOSITION}

Using the Stokes parameters we analyze the decomposition of arbitrary fields into a superposition of completely polarized and fully unpolarized components. This is the 3D analog of the well-known 2D decomposition. Although the following analysis is valid in classical as well as in quantum optics, we think that the quantum perspective throws some light on the problem. For example, it allows us to regard $\Phi$ as a one-photon density matrix.

Since $\Phi$ is Hermitian and non-negative, it can always be diagonalized for any field state,

$$
\Phi=\sum_{j=1}^{3} p_{j} v_{j} v_{j}^{\dagger}
$$

where the column vectors $v_{j}$ are the orthonormal eigenstates of $\Phi$ with eigenvalues $p_{j} \geqslant 0$. In quantum physics this is the diagonalization of the density matrix of a mixed state as a superposition of pure-state projectors $v_{j} v_{j}^{\dagger}$. On the other hand, in classical optics this represents a decomposition of the field in terms of mutually uncorrelated parts [18], where the weights $p_{j}$ represent field intensities.

The vectors $v_{j}$ provide a suitable resolution of the identity

$$
\sum_{j=1}^{3} v_{j} v_{j}^{\dagger}=I
$$

where $I$ is the $3 \times 3$ identity. This allows us to express one of the projectors - say, $v_{3} v_{3}^{\dagger}$-in terms of the other two and the identity. When this substitution is performed in Eq. (8.1) we get

$$
\Phi=\left(p_{1}-p_{3}\right) v_{1} v_{1}^{\dagger}+\left(p_{2}-p_{3}\right) v_{2} v_{2}^{\dagger}+p_{3} I
$$

It is worth noting that we should remove from Eq. (8.1) the vector with the least $p_{j}$, since otherwise the resulting weights for the remaining components in Eq. (8.3) would be negative, contrary to the interpretation as a superposition of uncorrelated fields. With this restriction the decomposition (8.3) becomes unique.

According to Eq. (2.5) the identity represents fully unpolarized light with $\left\langle S_{k}\right\rangle=0$, for $k=1, \ldots, 8$. Next we show that the $v_{j} v_{j}^{\dagger}$ components represent light with maximum degree of polarization.

To show this we notice that the normalization $v_{j}^{\dagger} \cdot v_{j}=1$ implies for $\Phi_{j}=v_{j} v_{j}^{\dagger}$ that $\Phi_{j}^{2}=\Phi_{j}$ and $\operatorname{tr} \Phi_{j}=1$. Moreover, from Eq. (2.5) we get

$$
\operatorname{tr} \Phi_{j}^{2}=\frac{1}{3}\left\langle S_{0}\right\rangle^{2}+\frac{1}{2} \sum_{k=1}^{8}\left\langle S_{k}\right\rangle^{2}, \quad \operatorname{tr} \Phi_{j}=\left\langle S_{0}\right\rangle .
$$

Therefore, the chain of equalities $\operatorname{tr} \Phi_{j}^{2}=\operatorname{tr} \Phi_{j}=1$ implies that the Stokes parameters corresponding to $\Phi_{j}$ satisfy

$$
\sum_{k=1}^{8}\left\langle S_{k}\right\rangle^{2}=\frac{4}{3}\left\langle S_{0}\right\rangle^{2}
$$

This is the condition for maximum degree of polarization if we use definition (2.7). If we use $P_{3 \mathrm{D}}$ in Eq. (2.8), we shall assume also that $\left\langle S_{0}^{2}\right\rangle=\left\langle S_{0}\right\rangle^{2}$ (the question of whether this requirement is satisfied or not cannot be answered with the information provided by the simple specification of $\Phi$ ).

\section{CONCLUSIONS}

We have carried out an analysis of 3D quantum polarization by using the Stokes operators. We have examined two definitions of the degree of polarization, showing their main properties and drawbacks.

We have found that the states with minimum polarization fluctuations and maximum degree of polarization are the SU(3) coherent states. We have shown that this is also approximately the case of the quadrature coherent states with large enough photon numbers, since they are Poissonian superpositions of $\mathrm{SU}(3)$ coherent states.

We have studied the relation between the 2D and 3D degrees of polarization by considering field states with a component in the vacuum state. It is shown that the embedding of two-dimensional fields into a three-dimensional framework is accompanied in general terms by an increase of the degree of polarization.

We have analyzed the decomposition of a threedimensional field into fully polarized and fully unpolarized mutually uncorrelated components. It is shown that, in the general case, the decomposition requires two polarized components uniquely determined.

We have applied this formalism to some relevant field states such as SU(3) coherent states, quadrature coherent states, and number states.

It is worth noting that we are examining polarization properties by using a small number of parameters, so that within the approach provided by the Stokes parameters very different states can possess the same polarization properties. This reflects the fact that there are situations where the Stokes parameters do not provide a complete picture of polarization. This is especially clear when all the Stokes parameters vanish since this can occur for field states that are not fully unpolarized light (for example, this is the case of the equipartitioned number states above). In other words, the Stokes parameters depend just on field correlations of the 
second order in the field amplitudes. In some particular cases, especially in the quantum domain, this is not enough and correlations of higher orders are necessary. This issue will be addressed elsewhere as a suitable generalization of the approach in Refs. [19].

\section{ACKNOWLEDGMENTS}

I thank Professor J. J. Gil for valuable comments and suggestions. This work has been supported by Project No. FIS2004-01814 of the Spanish Dirección General de Investigación del Ministerio de Educación y Ciencia.
[1] The Physics of Quantum Information, edited by D. Bouwmeester, A. Ekert, and A. Zeilinger (Sringer, Berlin, 2000); H. Paul, Introduction to Quantum Optics (Cambridge University Press, Cambridge, England, 2004).

[2] A. Luis and L. L. Sánchez-Soto, in Progress in Optics, edited by E. Wolf (Elsevier, Amsterdam, 2000), Vol. 41, p. 421.

[3] J. Pollet, O. Méplan, and C. Gignoux, J. Phys. A 28, 7287 (1995).

[4] J. J. Gil, J. M. Correas, P. A. Melero, and C. Ferreira (unpublished); J. J. Gil, J. M. Correas, C. Ferreira, I. San José, P. A. Melero, and J. Delso (unpublished).

[5] J. C. Samson and J. V. Olson, SIAM (Soc. Ind. Appl. Math.) J. Appl. Math. 40, 137 (1981).

[6] Ch. Brosseau, Fundamentals of Polarized Light: A Statistical Optics Approach (Wiley, New York, 1998).

[7] T. Carozzi, R. Karlsson, and J. Bergman, Phys. Rev. E 61, 2024 (2000).

[8] T. Setälä, A. Shevchenko, M. Kaivola, and A. T. Friberg, Phys. Rev. E 66, 016615 (2002).

[9] M. R. Dennis, J. Opt. A, Pure Appl. Opt. 6, S26 (2004); e-print physics/0309019.
[10] S. G. Schirmer, T. Zhang, and J. V. Leavy, J. Phys. A 37, 1389 (2004).

[11] R. D. Mota, M. A. Xicoténcatl, and V. D. Granados, J. Phys. A 37, 2835 (2004).

[12] A. P. Alodjants and S. M. Arakelian, J. Mod. Opt. 46, 475 (1999).

[13] R. Delbourgo, J. Phys. A 10, 1837 (1977).

[14] K. Nemoto, J. Phys. A 33, 3493 (2000); K. Nemoto and B. C. Sanders, ibid. 34, 2051 (2001).

[15] P. H. Moravek and D. W. Joseph, J. Math. Phys. 4, 1363 (1963).

[16] F. T. Arecchi, E. Courtens, R. Gilmore, and H. Thomas, Phys. Rev. A 6, 2211 (1972).

[17] P. W. Atkins and J. C. Dobson, Proc. R. Soc. London, Ser. A 321, 321 (1971); A. Luis and J. Peřina, Phys. Rev. A 53, 1886 (1996).

[18] P. Vahimaa and J. Tervo, J. Opt. A, Pure Appl. Opt. 6, S41 (2004).

[19] A. Luis, Phys. Rev. A 66, 013806 (2002); Opt. Commun. 216, 165 (2003); Phys. Rev. A 69, 023803 (2004). 